\title{
Mutations in KCNJ11, which encodes Kir6.2, are a common cause of diabetes diagnosed in the first 6 months of life, with the phenotype determined by genotype
}

Received: 3 November 2005 / Accepted: 28 February 2006 / Published online: 12 April 2006

C) Springer-Verlag 2006

\begin{abstract}
Aims/hypothesis: Heterozygous activating mutations in KCNJ11, which encodes the Kir6.2 subunit of the pancreatic ATP-sensitive potassium $\left(\mathrm{K}_{\mathrm{ATP}}\right)$ channel, cause both permanent and transient neonatal diabetes. A minority of patients also have neurological features. The identification of a $K C N J 11$ mutation has important therapeutic implications, as many patients can replace insulin injections with sulfonylurea tablets. We aimed to determine the age of presentation of patients with KCNJ11 mutations and to examine if there was a relationship between genotype and phenotype. Subjects and methods: KCNJ11 was sequenced in 239 unrelated patients from 21 countries, who were diagnosed with permanent diabetes before 2 years of age. Results: Thirty-one of the 120 patients (26\%) diagnosed in the first 26 weeks of life had a KCNJ11 mutation; no mutations were found in the 119 cases $(0 \%)$ diagnosed after this age. Fourteen different heterozygous mutations were identified, with the majority resulting from de novo mutations. These include seven novel mutations: H46Y, R50Q, G53D C166Y, K170T, L164P and Y330S. All 11 probands with the most common mutation, $\mathrm{R} 201 \mathrm{H}$, had isolated diabetes. In contrast, developmental delay in addition to diabetes was seen in four of five probands with the V59M mutation and two of four with the R201C mutation. Five patients with developmental delay, epilepsy and neonatal diabetes (DEND) syndrome had unique mutations not associated with other phenotypes. Conclusions/ interpretation: KCNJ11 mutations are a common cause of permanent diabetes diagnosed in the first 6 months and all patients diagnosed in this age group should be tested.
\end{abstract}

\footnotetext{
S. E. Flanagan · E. L. Edghill · A. L. Gloyn · S. Ellard

A. T. Hattersley $(\square)$

Institute of Biomedical and Clinical Science,

Peninsula Medical School,

Barrack Road,

Exeter, EX25DW, UK

e-mail: A.T.Hattersley@ex.ac.uk

Tel.: +44-1392-406806

Fax: +44-1392-406767
}

There is a strong genotype-phenotype relationship with the mutation being an important determinant of associated neurological features.

Keywords ATP-sensitive potassium channel - Diabetes · Genetics $\cdot$ KCNJ11 $\cdot$ Kir6.2 Permanent neonatal diabetes $\cdot$ Sulfonylureas

Abbreviations DEND: developmental delay, epilepsy and neonatal diabetes - I-DEND: intermediate DEND . ISPAD: International Society of Paediatric and Adolescent Diabetes $\cdot \mathrm{K}_{\mathrm{ATP}}$ : ATP-sensitive potassium $\cdot$ SUR1: sulfonylurea receptor 1

\section{Introduction}

The ATP-sensitive potassium $\left(\mathrm{K}_{\mathrm{ATP}}\right)$ channel plays a central role in glucose-stimulated insulin secretion from the pancreatic beta cell. The channel consists of two types of essential subunit: the pore-forming subunit Kir6.2 and the regulatory subunit sulfonylurea receptor 1 (SUR1), which is the target for sulfonylureas. Heterozygous activating mutations in $K C N J 11$, the gene for the Kir6.2 subunit, can cause both permanent and transient neonatal diabetes, which - in a minority of cases - have neurological features such as developmental delay and epilepsy [15]. The identification of a KCNJ11 mutation can have a major impact on a patient's treatment. Many patients have transferred from insulin injections to sulfonylurea tablets with an improvement in glycaemic control [1, 6-9], making the diagnosis of a KCNJ11 mutation clinically important.

The prevalence of diabetes due to a mutation in KCNJ11 is uncertain, with studies reporting mutations in between 34 and $64 \%$ of patients with permanent neonatal diabetes [1, $4,6,10]$. This may, in part, reflect differences in the definition of neonatal diabetes. Strictly speaking, the neonatal period is the first month of life; however, recent definitions of neonatal diabetes have included cases diagnosed before 3 months $[11,12]$. Massa et al. suggest 
that aetiological classifications do not follow this definition and propose the study of patients diagnosed in the first year as a new subgroup of permanent diabetes mellitus of infancy (PDMI) [4]. Only two studies to date have investigated patients aged over 6 months at diagnosis [4, 13], and the latter only looked at six patients diagnosed between the ages of 3 months and 1 year. More information is needed from large series of patients to determine the prevalence of KCNJ11 mutations in different age groups.

Initial reports suggest a possible relationship between the site of the mutation and the phenotype of the patient. The first report of activating KCNJ11 mutations [1] described three patients with severe neurological features (developmental delay and epilepsy) and neonatal diabetes, now known as the DEND syndrome $[5,14]$. These patients had mutations not seen in patients with isolated diabetes. Only three of 16 probands with a mutation at residue R201 had neurological symptoms, and all three patients had the less common R201C mutation $[1,4,6,7,9,10,13,15,16]$. Some patients with the V59M mutation have permanent neonatal diabetes with developmental delay but no epilepsy (intermediate DEND [I-DEND] syndrome) $[1,4,6,10]$, whereas others have no reported neurological features $[1$, 4]. Functional studies support a relationship between the site of the mutation and the ability of the channel to close in the presence of ATP. Disease severity is correlated with the extent of reduction in ATP sensitivity; Kir6.2 mutations associated with DEND syndrome are less sensitive to ATP than those that cause isolated diabetes $[14,17,18]$. The extent to which the clinical phenotype reflects the mutation requires further study.

We report the results of KCNJ11 sequencing in a consecutive series of 239 patients from 21 countries, who had diabetes diagnosed in infancy or early childhood. Studying this large cohort enabled us to gain better insight into the prevalence of $K C N J 11$ mutations at different ages of diagnosis and allowed further examination of genotypephenotype relationships.

\section{Subjects and methods}

Subjects We studied 239 patients with permanent diabetes (without remission) diagnosed before 2 years of age; $54 \%$ of the cohort were male. The majority were recruited following a request for referrals to the International Society of Paediatric and Adolescent Diabetes (ISPAD). The patients were recruited from 21 different countries across five continents (Fig. 1). Informed consent was obtained from all participants and the study was conducted in accordance with the Declaration of Helsinki as revised in 2000 .

KCNJ11 genetic analysis Genomic DNA was extracted from peripheral lymphocytes using standard procedures. The single exon of KCNJ11 was amplified in three fragments by PCR. All primers included a 5' M13 tail, and the sequences for fragments 1-3 were: $1 \mathrm{~F}$ 5'-CCG AGA GGA CTC TGC AGT GA-3', 1R 5'-TAG TCA CTT GGA CCT CAA TGG AG-3', 2F 5'-CTG CTG AGC CCT GTG TCA CC-3', 2R 5'-CAC GCC TTC CAG GAT GAC GAT3', 3F 5'-CTA CCA TGT CAT TGA TGC-3' and 3R 5'CCA CAT GGT CCG TGT GTA-3'. Sequencing was performed in both directions using universal M13 primers and a BigDye Terminator Cycler Sequencing Kit v1.1 (Applied Biosystems, Warrington, UK), and reactions were analysed on an ABI 3100 Capillary sequencer (Applied Biosystems). Sequences were compared to the published sequence (NM_000525.3) using Sequence Navigator (Applied Biosystems) or Staden analysis software (http://staden.sourceforge.net/, last accessed in February 2006). Novel mutations were tested for cosegregation with diabetes in other family members and in 200 normal chromosomes of UK white origin.
Fig. 1 World map showing the 21 countries of origin of patients studied. Numbers in brackets represent the total number of probands referred from each country

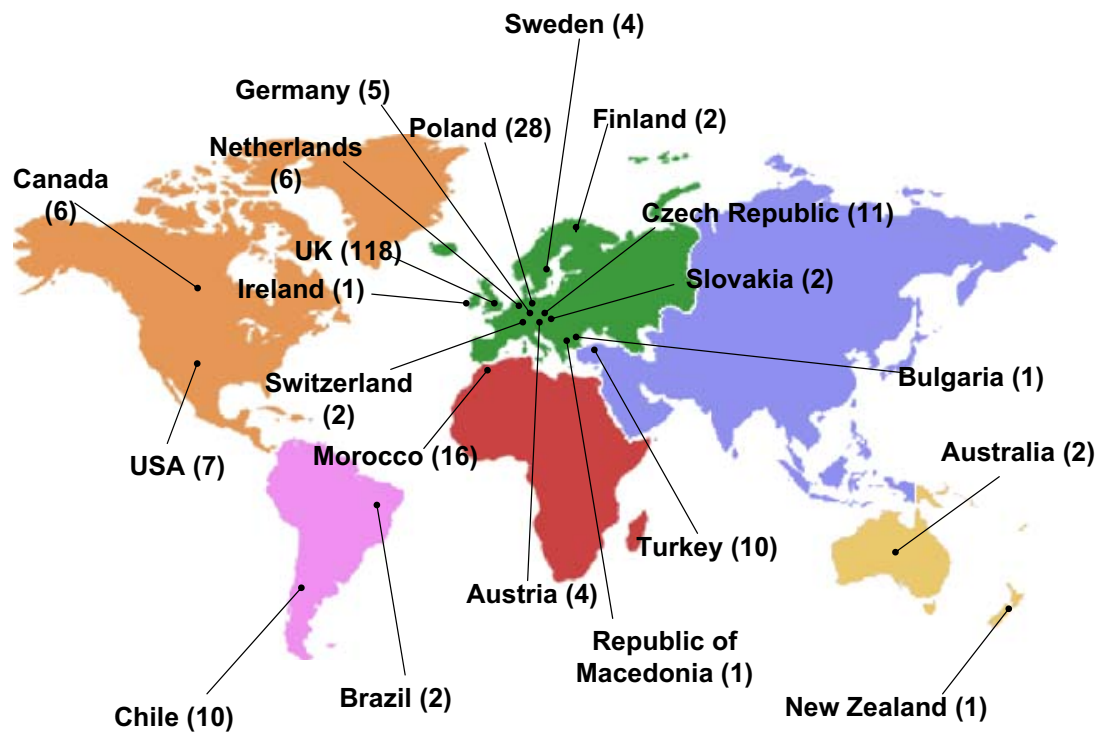


Microsatellite analysis Where possible, family relationships were confirmed using a combination of the following six microsatellites on chromosome 11: D11S902, D11S419, D11S1397, D11S1901, D11S921 and D11S1888.

Clinical studies All patients underwent routine developmental assessment by a trained paediatrician. The assessment nearest to the age at the time of the testing for Kir6.2 was used for classification. A paediatric neurologist confirmed any abnormal neurological features. The following definitions were used:

1. Developmental delay - this was a marked delay of motor, language and cognitive abilities such that functional age in two or all of these parameters was at least $25 \%$ lower than the child's chronological age.

2. Muscle weakness - clear objective evidence of symmetrical muscle weakness, usually involving the legs.

3. Epilepsy-clinically observed seizures with generalised abnormal activity on an EEG.These features were only used in the classification of patients' KCNJ11 disease if there was not an alternative explanation (e.g. severe cerebral oedema following the treatment of diabetic ketoacidosis). Patients were classified into full DEND syndrome when the patient had severe developmental delay (functional age $<50 \%$ chronological age) and generalised epilepsy diagnosed in the first 12 months of life, and I-DEND syndrome when there was developmental delay but the functional age was $>50 \%$ of chronological age and the patients did not have epilepsy.

Locally measured $\mathrm{HbA}_{1 \mathrm{c}}$ results were requested from the physician at the time of referral. All assays were said to be DCCT aligned, but precise data on normal reference ranges and methods of analysis cannot be given because of the many different laboratories involved.
Fig. 2 a Illustration of the four Kir6.2 subunits of the channel, showing the 14 mutated residues. The illustration is based on the crystal structure of the potassium channel KirBac1.1 [25]. Each of the four subunits is represented by a different coloured ribbon structure (red, blue, lime, green). The mutated residues in patients with neonatal diabetes are shown in yellow. Residues have been labelled and colour coded according to the region of the protein in which they are located (orange text, residues located in the ATP binding site; grey text, residues located in the slide helix; green text, residue located in the cytosolic $\mathrm{NH}_{2}$ terminal domain; black text, residue located in the domain involved in the interaction of the Kir6.2 and SUR1 subunits; purple text, residues lying in the outer transmembrane helix; and blue text, residue located in the permeation pathway at the mouth of the transmembrane pore). The shadowed band represents the cell membrane and the labels 'inside' and 'outside' refer to areas outside and inside the cell. b Schematic representation of KCNJ11 showing the 14 mutations identified in 31 unrelated probands. Numbers in brackets represent the total number of probands found to carry the mutation. The seven novel mutations are shown in grey italic print a

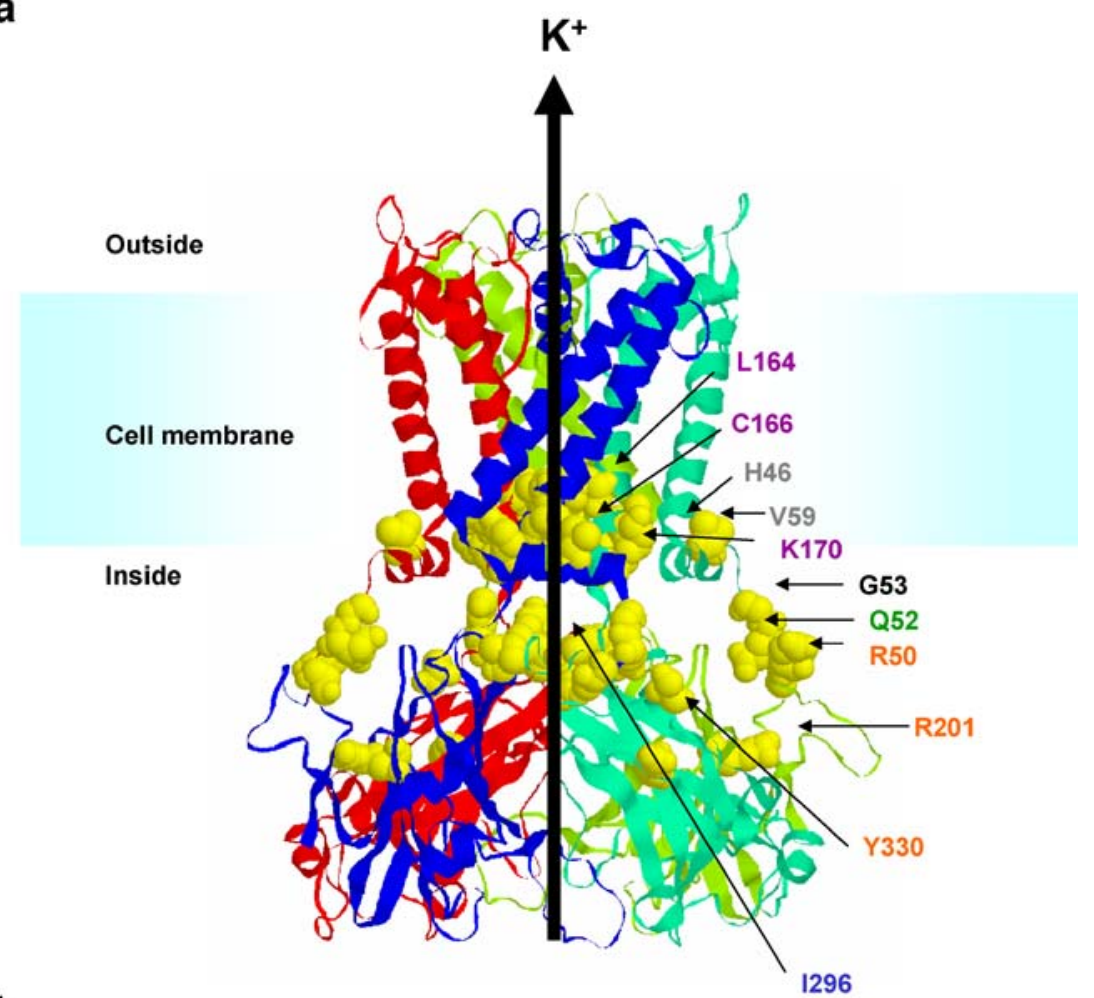

b

\section{KCNJ11}

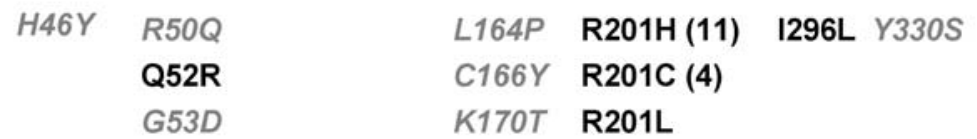

V59M (5)

V59G 


\section{Results}

Heterozygous activating mutations were identified in 31 of 239 probands with permanent diabetes (Fig. 2). Fourteen of these families have previously been reported $[1,2,7,9,13$, 15]. The majority of probands with a KCNJ11 mutation were white $(n=24)$, but mutations were also found in patients of the following ethnicities: Asian $(n=2)$, black African $(n=1)$, Hispanic $(n=1)$, White/African American $(n=1)$, Hispanic/Asian $(n=1)$, Black African/White/ American Indian $(n=1)$. We found 14 different missense mutations (Fig. 2), of which seven are novel: H46Y (c.136C $>$ T), R50Q (c.149G $>$ A), G53D (c.158G $>A$ ), L164P (c.491T $>$ C), C166Y (c.497G $>$ A), K170T (c.509A >C), Y330S (c.989A >C c.990C $>$ T). These novel mutations were not found in 200 normal chromosomes, and within families these mutations were only present in subjects with permanent, young-onset diabetes. All the mutations affect residues that are conserved in dogs, mice, rats, chickens, Fugu and zebrafish. Three mutations occurred in more than one proband, namely, $\mathrm{R} 201 \mathrm{H}$ $(n=11), \mathrm{R} 201 \mathrm{C}(n=4)$ and V59M $(n=5)$.

In all 31 families there was an affected proband born to two unaffected parents, although in four families diabetes was then transmitted to a child or children in the next generation. No families had more than two affected generations. DNA samples were available from both unaffected parents of 22 probands allowing us to establish that these were de novo mutations, as the mutations were not present in the parents, and microsatellite analysis confirmed the family relationships.

The prevalence of KCNJ11 mutations was dependent upon the age at diagnosis (Fig. 3). All subjects with a $K C N J 11$ mutation were diagnosed with diabetes at the age of 6 months or under; the median age of diagnosis was 5 weeks. The detection rate was $26 \%$ before 6 months of age and $0 \%$ after. Patients with KCNJ11 mutations were more commonly diagnosed with diabetes in the first 3 months than in the second 3 months ( 24 vs 7 ), but the percentage of cases that had a mutation was similar (24 vs $39 \%, p=0.17$ ) in the two age groups. The age at diagnosis for one previously reported patient (I296L, ISPAD 43 [1]) has changed, as further clinical information showed that analysis of glucose in a blood spot used for screening for congenital hypothyroidism on day 7 was $20 \mathrm{mmol} / \mathrm{l}$, which is diagnostic of diabetes.

The clinical characteristics of the probands and the six additional affected family members with KCNJ11 mutations are shown in Table 1 . In all patients diabetes was treated with insulin and most required doses consistent with a full replacement dose. Low birthweight was a consistent feature, with the median birthweight being the third centile and $67 \%$ being classified as small for gestational age as their weights were below the 10th centile.

In addition to diabetes, a spectrum of neurological features were present in 11 of $37(30 \%)$ patients with a KCNJ11 mutation. Five of these patients (with the Q52R, G53D, V59G, C166Y and I296L mutations) had the DEND

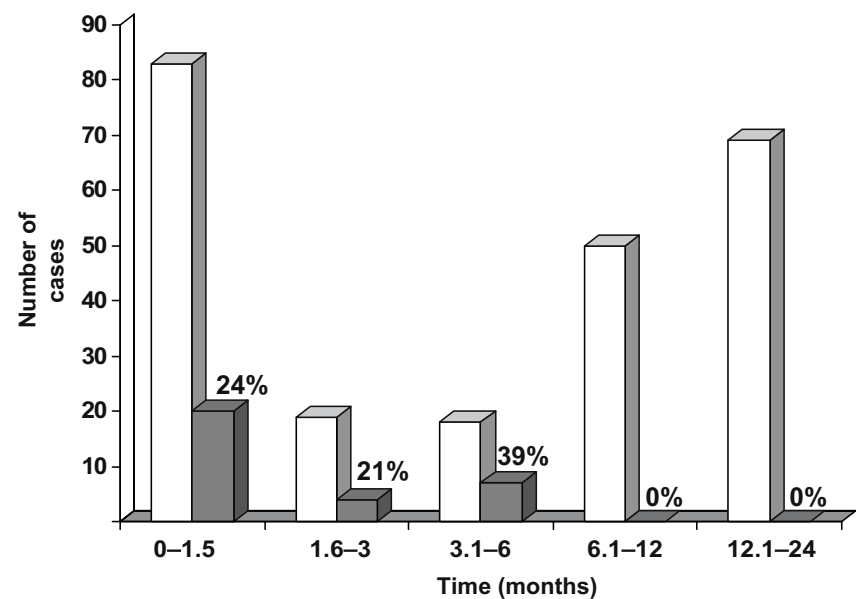

Fig. 3 Frequency of KCNJ11 mutations in probands grouped by age at diagnosis. The number of patients tested is shown in the white columns, with age of diagnosis grouped from 0 to 2 years. The number of KCNJ11 mutations identified within each group is shown by the grey columns and the percentage of positive tests is stated above

syndrome with all three features of developmental delay, epilepsy and neonatal diabetes, two of which are previously unreported. The first unreported case (C166Y) had profound developmental delay and microcephaly and had been diagnosed with West syndrome at 3 months of age. The second case (G53D) had developmental delay and seizures during infancy and an abnormal EEG. In addition, there were two patients with developmental delay (both patients had the R201C mutation) and four patients with developmental delay and muscle weakness (all four patients had the V59M mutation), but none of these six patients had epilepsy. We describe this as I-DEND

Table 1 Clinical and biochemical characteristics of the 37 patients with $K C N J 11$ mutations from 31 families

\begin{tabular}{ll}
\hline Characteristic & Value \\
\hline Sex & $62 \%$ male \\
Age at diagnosis diabetes (weeks) & $5(0-26)$ \\
Age when tested for KCNJ11 & $10(0.02-56)$ \\
mutations (years) & \\
Most recent dose of insulin (U/kg) & $0.7(0.25-2.0)$ \\
HbA $_{1 \mathrm{c}}(\%)$ & $7.9(6.0-18.0)$ \\
Birthweight (g) & $2,548(1,440-3,500)$ \\
Gestational age (weeks) & $39(32-42)$ \\
Centile birthweight & $3(<1-58)$ \\
Proportion SGA & $67 \%$ \\
(birthweight $<10$ th centile) & \\
Any neurological features & $11 / 37(30 \%)$ \\
Developmental delay & $11 / 11$ \\
Muscle weakness & $9 / 11$ \\
Epilepsy & $5 / 11$ \\
\hline
\end{tabular}

Median results are shown, with the range given in brackets $S G A$ Small for gestational age

${ }^{a}$ Centile birthweights were calculated according to UK growth charts [24] 
syndrome [5]. The patients with neurological features did not have a more severe beta cell phenotype, as shown by birthweight $(2,550 \mathrm{vs} 2,548 \mathrm{~g}, p=0.63)$ and age at diagnosis ( 5 vs 6 weeks, $p=0.82$ ).

We found evidence to support a genotype-phenotype relationship for the common KCNJ11 mutations (see Table 2). The most common mutation, $\mathrm{R} 201 \mathrm{H}$, was associated with isolated diabetes in all of the 14 patients, in contrast to V59M where four of five patients had neurological features (I-DEND syndrome) $(p=<0.001)$. Two of the seven patients (both probands) with the R201C mutation had developmental delay in addition to diabetes.

\section{Discussion}

We report on 31 probands with 14 different heterozygous activating mutations in $K C N J 11$, identified by mutation screening 239 patients with permanent diabetes before the age of 2 years. The detection of KCNJ11 mutations is increasingly important, as many cases have been able to discontinue insulin injections and achieve better glycaemic control on sulfonylurea tablets $[1,6-9]$. We show that in this large series, mutations in this gene are only detected in those diagnosed with diabetes in the first 6 months of life, and provide further evidence for a genotype-phenotype relationship.

We describe seven novel mutations (H46Y, R50Q, G53D, L164P, C166Y, K170T, Y330S). Although functional studies have not been performed for all of these, we are confident that these mutations are pathogenic since (1) none of the mutations were found in 200 normal chromosomes; (2) all seven residues are conserved in other species; and (3) all seven mutations were shown to be spontaneous mutations. In five of the seven cases, different mutations at the same amino acid have previously been reported: (R50P [4], G53S[2], G53R[2], G53N[10], K170R [4], K170N [4], C166F [19], Y330C [6, 10]). The finding of multiple mutations at the same codon highlights the functional importance of these residues. Functional studies have already established an altered response to ATP by $\mathrm{K}_{\text {ATP }}$ channels with mutations in Kir6.2 at the following residues: R50, Q52, G53, V59, R201, L164, C166 or I296 [1, 2, 14, 17, 18, 20, 21].

In all families there was an affected patient with a KCNJ11 mutation whose parents were unaffected. This is consistent with a spontaneous mutation in the affected patient. Where DNA was available from both parents $(n=22)$, we were able to confirm a de novo mutation. The high prevalence of spontaneous mutations is consistent with other series where familial cases were rare or not found $[4,6,10]$. We have previously reported a family in which the unaffected father of two half-siblings with a heterozygous KCNJ11 mutation showed no evidence of the mutation in leukocyte DNA [2], suggesting that he was a germline mosaic carrier of the mutation. The possibility of germline mosaicism means that the risk of a further sibling being affected is significantly higher than the very low chance of a second spontaneous mutation.
In this large series, mutations in $K C N J 11$ were found exclusively in those diagnosed with diabetes within the first 6 months of life. Interestingly, although patients were more likely to have been diagnosed in the first 3 months (Fig. 2), the percentage of cases that had a mutation was similar in those diagnosed in the first 3 months $(24 \%)$ and in those diagnosed between 3 and 6 months (39\%). Only those diagnosed in the first 3 months have previously been classified as having neonatal diabetes, and this result confirms that a broader definition is needed when considering KCNJ11 mutations [4]. A cut-off point at 6 months is consistent with previous work looking at autoantibodies and HLA suggesting that type 1 diabetes is rare in those aged below 6 months [22]. Our study would not support testing for KCNJ11 mutations in patients diagnosed after 6 months, although relatives of probands with transient neonatal diabetes have been diagnosed at 3 , 5,22 and 26 years $[2,3]$. The detection rate of $26 \%$ in our series was lower than the $34-64 \%$ previously reported $[1$, $4,6,10]$, although the $95 \%$ confidence limits of all series overlap. This may be because in this international series, detailed phenotypic assessment was not performed on a longitudinal basis before testing. Hence, patients who remitted after DNA was analysed will not have been excluded, and phenotypic selection is likely to be stricter in the long-term longitudinal series established in France and Italy $[4,10]$.

We have identified 11 patients who have neurological features as well as permanent diabetes. This provides further evidence that some patients with a KCNJ11 mutation have neurological features as a result of the mutated $K_{\text {ATP }}$ channel. Five patients have full DEND syndrome with the key characteristics of developmental delay, epilepsy and neonatal diabetes. There is a sixth French patient with severe neurological deficit and generalised epilepsy with the characteristics of West syndrome [19]. Functional studies have suggested that the mutations associated with DEND syndrome are more severe, with the channel less likely to close in the presence of ATP compared with mutations associated with isolated neonatal diabetes [14]. The other six patients have the I-DEND syndrome (neonatal diabetes with moderate developmental delay and/or muscle weakness but not epilepsy), bringing the total number of reported cases of this phenotype to $14[4,6,10]$. The most common mutation associated with I-DEND syndrome is V59M (ten of 15 cases), and functional studies have shown that the V59G mutation found in a patient with DEND syndrome has a more severe effect than V59M [23].

Our series provides further evidence for a genotypephenotype relationship for KCNJ11 mutations. The most common mutation, $\mathrm{R} 201 \mathrm{H}$, has now been reported in 20 patients $[6,8,10,16]$, with none of the patients having neurological features. In contrast, of the 13 patients with the V59M mutation, ten $(77 \%)$ have intermediate DEND syndrome $[1,4,6,10]$. The mutations causing the full DEND syndrome have not been described in patients with isolated diabetes. It is interesting that three of nine (33\%) patients with the R201C mutation have intermediate 


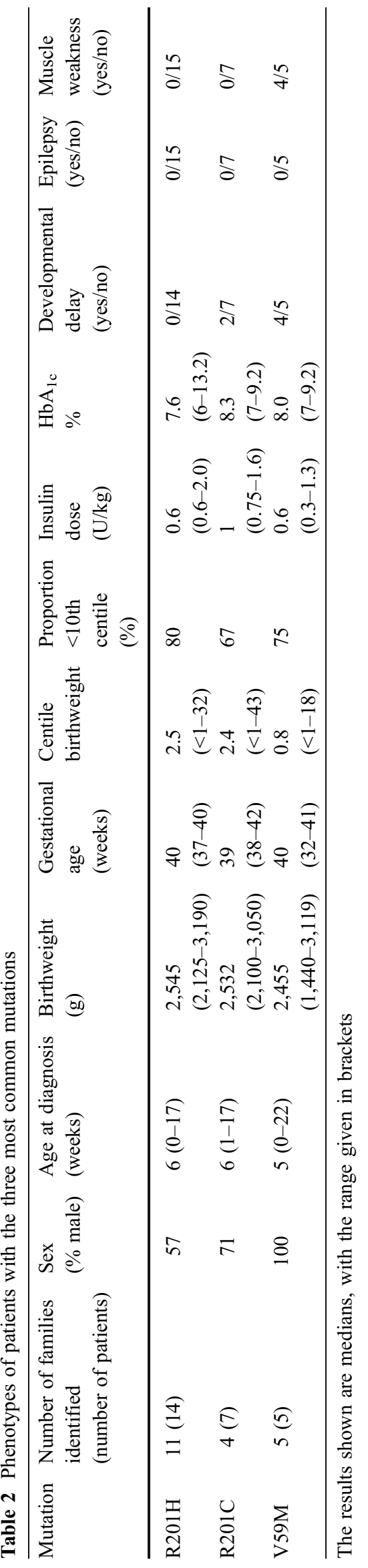


DEND $[4,10,13,15]$, which is not seen in those with the $\mathrm{R} 201 \mathrm{H}(0$ of 20$)$ mutation at the same residue. This has only become clear with larger numbers and is in keeping with the greater severity of mutation seen in vitro [23]. It is interesting that the V59M and R201C mutations are not associated with a consistent neurological phenotype. This may, in part, reflect differences in assessment (e.g. developmental delay is difficult to detect in the first year of life), but it also is likely that there is phenotypic variation between subjects with the same mutation. Variation in severity of beta cell dysfunction has been described in subjects with the R201H mutation $[1,7,16]$.Therefore, no genotype-phenotype relationship is absolute and there must be modification by other genetic and/or environmental factors.

Localisation of the mutated residues in structural models of the channel show that residues associated with isolated diabetes (R50, L164, R201, Y330) cluster around the ATP binding site $[17,20]$, thus decreasing ATP sensitivity by reducing ATP binding. Residues associated with the more severe phenotype (Q52, G53, V59, C166, I296) frequently occur at a distance from the ATP binding site, either within the pore-forming domain or the slide helix area of the protein $[2,14,17,20]$. Functional analysis of these mutations shows that the mutated residue within the Kir6.2 protein correlates with the observed phenotype $[14,17]$. All mutations causing permanent diabetes, tested to date, have been shown to lower the sensitivity of the channel to ATP, reducing the channel's ability to close in response to elevated levels of ATP. The mutations associated with DEND syndrome show the most profound reduction in response to ATP, with mutations causing isolated permanent diabetes, having a reduced response compared with those associated with transient diabetes [2, $14,17]$.

Our study does have limitations. First, we did not test any patients diagnosed over the age of 2 years, and therefore we cannot make conclusions about this age group. Secondly, developmental delay was not assessed by formal criteria at a set age by a single physician as a result of the age distribution and international location of patients. This is likely to have resulted in a lack of clinical detection of some of the neurological characteristics or inconsistent reporting, especially as those patients thought not to have problems will not have been referred to specialist neurologists. Patients with KCNJ11 mutations were aged from 6 days to 56 years old at the time of this study. In younger patients, the neurological phenotype may not have been clinically detectable. The third limitation is that some of the younger patients may not have had 'permanent' diabetes: although they were diabetic when the study was performed they were still young ( $<6$ months) and may remit later and so have transient diabetes. The final limitation is that this study is a referred series rather than an epidemiological sample, so those sent for screening may be subject to selection bias. Population-based studies would establish if this were the case.

In conclusion, we have shown that the majority of patients with mutations in KCNJ11 are diagnosed with diabetes before the age of 6 months but are not confined to those with a formal diagnosis of neonatal diabetes. We provide further evidence for discrete neurological phenotypes (DEND syndrome and I-DEND syndrome) as a consequence of a mutated Kir6.2 channel. Our work supports a clear genotype-phenotype relationship, supporting the hypothesis that the phenotype observed is predominantly related to the nature of the mutation within the protein. The major therapeutic implications for those found to have a KCNJ11 mutation highlights the importance of screening all patients who are diagnosed with permanent diabetes in the first 6 months of life. Our study does not support routine testing after this age.

Acknowledgement The authors would like to thank all of the families for their participation in this study, the many referring clinicians and UK MODY Link Nurses for helping to make this study possible. Our thanks to E. Pearson for his clinical expertise, K. Stals for her technical help and B. Shields for statistical analysis. We thank the Wellcome Trust for financial support. A. T. Hattersley is a Wellcome Trust Research Leave Fellow.

Duality of interest The authors are not aware of any duality of interest.

\section{References}

1. Gloyn AL, Pearson ER, Antcliff JF et al (2004) Activating mutations in the gene encoding the ATP-sensitive potassiumchannel subunit Kir6.2 and permanent neonatal diabetes. N Engl J Med 350:1838-1849

2. Gloyn AL, Reimann F, Girard C et al (2005) Relapsing diabetes can result from moderately activating mutations in KCNJ11. Hum Mol Genet 14:925-934

3. Yorifuji T, Nagashima K, Kurokawa K et al (2005) The C42R mutation in the Kir6.2 (KCNJ11) gene as a cause of transient neonatal diabetes, childhood diabetes, or later-onset, apparently type 2 diabetes mellitus. J Clin Endocrinol Metab 90:3174-3178

4. Massa O, Iafusco D, D'Amato E et al (2005) KCNJ11 activating mutations in Italian patients with permanent neonatal diabetes. Hum Mutat 25:22-127

5. Hattersley AT, Ashcroft FM (2005) Activating mutations in Kir6.2 and neonatal diabetes: new clinical syndromes, new scientific insights, and new therapy. Diabetes 54:2503-2513

6. Sagen JV, Raeder H, Hathout E et al (2004) Permanent neonatal diabetes due to mutations in KCNJ11 encoding Kir6.2: patient characteristics and initial response to sulfonylurea therapy. Diabetes 53:2713-2718

7. Klupa T, Edghill EL, Nazim J et al (2005) The identification of a R201H mutation in KCNJ11, which encodes Kir6.2, and successful transfer to sustained-release sulphonylurea therapy in a subject with neonatal diabetes: evidence for heterogeneity of beta cell function among carriers of the R201H mutation. Diabetologia 48:1029-1031

8. Zung A, Glaser B, Nimri R, Zadik Z (2004) Glibenclamide treatment in permanent neonatal diabetes mellitus due to an activating mutation in Kir6.2. J Clin Endocrinol Metab 89:5504-5507

9. Codner E, Flanagan S, Ellard S, Garcia H, Hattersley AT (2005) High-dose glibenclamide can replace insulin therapy despite transitory diarrhea in early-onset diabetes caused by a novel R201L Kir6.2 mutation. Diabetes Care 28:758-759

10. Vaxillaire M, Populaire C, Busiah K et al (2004) Kir6.2 mutations are a common cause of permanent neonatal diabetes in a large cohort of French patients. Diabetes 53:2719-2722 
11. Shield JPH (1996) Neonatal diabetes. In: Shield JPH, Baum JD (eds) Childhood diabetes. Bailliere Tindall, London, pp 681-740

12. Polak M, Shield J (2004) Neonatal and very-early-onset diabetes mellitus. Semin Neonatol 9:59-65

13. Edghill EL, Gloyn AL, Gillespie KM et al (2004) Activating mutations in the KCNJ11 gene encoding the ATP-sensitive $\mathrm{K}+$ channel subunit Kir6.2 are rare in clinically defined type 1 diabetes diagnosed before 2 years. Diabetes 53:2998-3001

14. Proks P, Girard C, Haider S et al (2005) A gating mutation at the internal mouth of the Kir6.2 pore is associated with DEND syndrome. EMBO Rep 6:470-475

15. Gloyn AL, Cummings EA, Edghill EL et al (2004) Permanent neonatal diabetes due to paternal germline mosaicism for an activating mutation of the KCNJ11 gene encoding the Kir6.2 subunit of the beta-cell potassium adenosine triphosphate channel. J Clin Endocrinol Metab 89:3932-3935

16. Colombo C, Delvecchio M, Zecchino C, Faienza MF, Cavallo L, Barbetti F (2005) Transient neonatal diabetes mellitus is associated with a recurrent (R201H) KCNJ11 (KIR6.2) mutation. Diabetologia 48:2439-2441

17. Proks P, Antcliff JF, Lippiat J, Gloyn AL, Hattersley AT, Ashcroft FM (2004) Molecular basis of Kir6.2 mutations associated with neonatal diabetes or neonatal diabetes plus neurological features. Proc Natl Acad Sci USA 101:17539-17544
18. Antcliff JF, Haider S, Proks P, Sansom MS, Ashcroft FM (2005) Functional analysis of a structural model of the ATPbinding site of the KATP channel Kir6.2 subunit. EMBO J $24: 229-239$

19. Gloyn AL, Diatloff-Zito C, Edghill EL et al (2006) Extrapancreatic manifestations of KCNJ11 activating mutations: evidence to support a discrete syndrome of developmental delay, epilepsy and neonatal diabetes (DEND). Eur J Hum Genet (in press)

20. Haider S, Antcliff JF, Proks P, Sansom MS, Ashcroft FM (2005) Focus on Kir6.2: a key component of the ATP-sensitive potassium channel. J Mol Cell Cardiol 38:927-936

21. Tucker SJ, Gribble FM, Proks P et al (1998) Molecular determinants of KATP channel inhibition by ATP. EMBO J 17:3290-3296

22. Iafusco D, Stazi MA, Cotichini R et al (2002) Permanent diabetes mellitus in the first year of life. Diabetologia 45:798-804

23. Proks P, Girard C, Ashcroft FM (2005) Functional effects of KCNJ11 mutations causing neonatal diabetes: enhanced activation by MgATP. Hum Mol Genet 14:2717-2726

24. Cole TJ, Freeman JV, Preece MA (1998) British 1990 growth reference centiles for weight, height, body mass index and head circumference fitted by maximum penalized likelihood. Stat Med 17:407-429

25. Kuo A, Antcliff JF, Rahman T (2003) Crystal structure of the potassium channel KirBac 1.1 in the closed state. Science 300:1922-1926 